\title{
Fault populations and their relationship to the scaling of surface roughness
}

\author{
G. Pickering, ${ }^{1}$ J. M. Bull, and D. J. Sanderson \\ School of Ocean and Earth Science, Southampton Oceanography Center \\ University of Southampton, Southampton, England
}

\begin{abstract}
The relationship between the scaling properties of faulted geological surfaces and parameters describing the underlying fault population are investigated using simulations of a dip-slip faulted surface. Analysis of multiple simulations of sections through the surface allowed the construction of a statistical relationship between the parameters defining the fault population and the fractal dimension of the surface. The results indicate a direct, if complex, relationship between the fault population and the scaling of the surface roughness. The main determining factor is the displacement distribution, with spacing and dip having only a minor contribution. This relationship is tested against examples from the Moray Firth, Scotland, and the central Indian Ocean.
\end{abstract}

\section{Introduction}

Natural topographies have generally been shown to be selfaffine fractals by studies of exposed rock surfaces [e.g. Mandelbrot, 1977; Brown and Scholz, 1985; Farr, 1992; Huang and Turcotte, 1989]. A self-affine fractal is anisotropic; one co-ordinate scales differently from the other, so that in two dimensions ( $\mathrm{rx}, \mathrm{rHy}$ ) is statistically similar to $(x, y)$, where $H$ is the Hurst exponent and $r$ is the scaling factor. The anisotropy inherent with self-affine fractals means that care must be taken when selecting measurement methods for the fractal dimension: techniques such as the length estimator or roughness scaling, as described later, may be used; box-counting and ruler methods are inappropriate (see Malinverno [1995], for more discussion).

Many authors have investigated the fractal properties of topographic sections, from the continents to ocean floor topography (see Turcotte [1992] and Malinverno [1995] for reviews). Less work has been directed at relating these properties to the processes responsible for their formation. King [1983] proposed two main processes which might result in a fractal topography; faulting and erosion. In this paper consideration is given to the contribution of faulting and more particularly to the relationship between dip-slip fault populations and the roughness of the faulted surface they produce.

Deriving this relationship has both fundamental and practical value. Linking the observable geometries to geological processes provides a basis for interpreting such processes. On a more prosaic level, measurements of the fractal properties of surfaces are more easily obtained than underlying fault displacements, which are based on interpretation and measurement of individual faults. The description of the fault population that can then be derived has particular value in predicting sub-resolution faulting for both crustal extension estimates and other applications within the petroleum and mining industries [Childs et al., 1990; Scholz and Cowie, 1990; Pickering et al., 1995, 1997].

'Now at Ikoda Limited, Twickenham, England, United Kingdom.

Copyright 1999 by the American Geophysical Union.
The most detailed previous study of surface roughness due to faulting was by Malinverno and Cowie [1993]. Their approach considered a simple model of vertical faults offsetting an elastic plate, overlying an inviscid fluid. The expected topography was calculated analytically, using a mechanical model of the response of the plate to the faulting. Their aim was to quantify the contribution of normal faulting to the roughness of bathymetric profiles across mid-ocean ridges. They assumed the fault displacement population was negative exponential, as there is some evidence that fault populations at mid-ocean ridges follow such a distribution [Cowie et al., 1994]. Their analysis is not directly applicable to continental and non ridge related oceanic fault displacement populations, as these are typically power law distributed [e.g. Kakimi, 1980; Childs et al., 1290; Walsh et al., 1991; Bull and Scrutton, 1992; Jackson and Sanderson, 1992; Pickering et al., 1994], particularly in the case of normal fault and reverse fault populations, which are the topic of this paper. Moreover, it is unlikely that their relatively simple elastic model is applicable to more complex continental tectonics.

In this paper, the faulted horizon is modeled as a horizontal surface offset by faults, without including the flexural response due to the faulting. This approximation is reasonable for two reasons. First, it is unlikely that there is any significant flexural response for normal or reverse faults with throws significantly less than the crustal thickness, as these are highly likely to be intra-crustal. Second, the flexural response of the largest faults, which may penetrate through the crust, will not contribute to the roughness measurements of the surfaces except at the largest length scales, due to the relatively long wavelength of such a response. As the bulk of the measurements made are at small length scales, the comparison between the model sections and the natural sections will not be compromised.

A simulation was made in which a series of faulted sections were analyzed to find the average roughness due to a particular fault population. The advantage of this technique is that the simplifications needed for an analytical solution can be avoided, allowing investigation of complex geometries. In this work, sections rather than surfaces were used as (1) the fractal dimension of a section $\left(F_{I D}\right)$ is simply related to the dimension of the surface $F_{2 D}$ by: 
[e.g. Feder, 1988] and (2) fault populations are routinely measured using sections and the parameters derived can be easily related to the fault population in higher dimensions [Marrett and Allmendinger, 1991].

The relationship between the roughness of the section and the fault population was derived by changing the parameters describing the population and measuring the fractal dimension of the resulting section. Before outlining the simulation, the analysis methods used to measure the fractal dimension of a self-affine curve are described. The simulation is then discussed in detail together with the results of tests designed to find which parameters are most important in determining the roughness of the horizon. The simulation is then compared to the results of analyses of faulted horizons derived from seismic sections from the Moray Firth, NE Scotland, and the Indian Ocean.

\section{Methods of Data Analysis}

The first step in the fractal analysis of self-affine profiles is to sample the profile at regular intervals to produce a series. The three most common analysis methods applied to these series are (1) roughness scaling methods, (2) scaling of a length estimator, and (3) spectral analysis. In this paper, only the first and second methods are used due to difficulties in applying the spectral techniques to this particular problem, as discussed later.

\subsection{Roughness scaling methods}

These methods are based on the relationship between the average dispersion of a sub set of a series and the size of each sub-set. For a time series this dispersion is usually defined as the root-mean-square (rms) deviation of the members of the subset from the mean. The rms deviation $(\sigma)$ is a measure of the roughness of the profile. The size of the subset is the number of values multiplied by the sample interval; for topographical sections this corresponds to the horizontal length $(l)$. For a self-affine fractal:

$$
\sigma=\gamma l^{H}
$$

where $H$ is the Hurst exponent [Mandelbrot, 1977] and $\gamma$ is a constant. $F$ is simply related to $H$ by [Mandelbrot, 1985]:

$$
F=2 \cdot H
$$

There are different methods of employing equation 3 to find the fractal dimension of a profile or time series. The simplest is that recently described by Ivanov [1994]; referred to as the "scaling of internal dispersion". The series is divided into sets of equal length, and the rms deviation from the mean $(\sigma)$ is calculated for each one. These values are then averaged to give $<\sigma\rangle$. By varying the length of these subsets, the relationship between $\langle\sigma\rangle$ and length can be derived, giving the value of $H$ and $F\left(F_{l}\right)$. A similar method was employed by Malinverno and Cowie (1993) to calculate $F_{M C}$, where rather than the deviation from the mean, the deviation from a line fit using a least squares method was calculated. The two measures of dispersion are similar; however they do give somewhat different results, as will be discussed later.

\subsection{Length estimator method}

This method was developed by Higuchi [1988] and has been applied to various geological problems [Schulz et al., 1994]. Consider a time-series $X$ of $N$ values;

$$
X(1), X(2), X(3), \ldots, X(N)
$$

new time series are constructed from $X$, defined as follows:

$$
\begin{aligned}
& X_{k}^{m}: X(m), X(m+k), X(m+2 k), \ldots, \\
& X\left(m+\left[\frac{N-m}{k}\right] \bullet k\right)(m=1,2, \ldots, k)
\end{aligned}
$$

where brackets denotes integer notation, and both $k$ and $m$ are integers. Variable $m$ gives the initial time for each sub series, $k$ is the interval. For any given $k$, there are $k$ sets of new timeseries. For example, if $k=3$ and $N=100$, then three series are obtained:

$$
\begin{gathered}
X_{3}^{\prime}: X(1), X(4), X(7), \ldots ., X(97), X(100) \\
X_{3}^{2}: X(2), X(5), X(8), \ldots, X(98) \\
X_{3}^{3}: X(3), X(6), X(9), \ldots, X(99)
\end{gathered}
$$

The length of the curve $X_{m}^{k}$ is defined as follows:

$$
L_{m}(k)=\left(\begin{array}{l}
{[. .]} \\
\sum_{i=I}|X(m+i k)-X(m+(i-1) . k)|
\end{array}\right) \frac{N-1}{[. .] \cdot k}
$$

where $(N-1) /[.] .$.$k is the normalisation factor and:$

$$
[. .]=\left[\frac{N-m}{k}\right]
$$

The length of the curve for the time interval $k$ is defined as the average value over $k$ sets of $L_{m}(k),(<L(k)>)$. If the time series represent a fractal curve then:

$$
<L(k)>\propto k^{(f-1)}
$$

where $F$ is the fractal dimension $\left(F_{L E}\right)$. Graphs of $\langle L(k)>k$ are usually plotted against $k$, as these yield $F$ directly. Note that for a series where the sample interval is $\neq 1, k$ is the number of sample intervals rather than the size of the analysis interval. Higuchi [1988] demonstrates that the method is a significant improvement on other curve-length estimator methods [e.g. Burlaga and Klein, 1986] and it gives the expected results on simulated data with known fractal dimension. The method is similar to the scaling of "structure functions" used by Weissel et al. [1994].

\subsection{Spectral Analysis}

This is the most common technique applied in the literature to measure the scaling properties of series. A Fourier transform of the series is taken, producing an amplitude spectrum. For a fractal curve this amplitude spectrum should have the form [Berry and Lewis, 1980]:

$$
A \cong f^{-(5 / 2-f)}
$$

where $A$ is the amplitude at frequency $f$ and $F$ is the fractal dimension. The exponent of the amplitude spectrum is commonly referred to as $b$, therefore $b=-(5 / 2-F)$.

This simple relationship can only ever be an approximation. The fractal dimension $F$ for a two dimensional profile must lie between 1 and 2 by definition [Mandelbrot, 1977]. This corresponds to $-3 / 2 \leq b \leq-1 / 2$. However, it is possible to produce a profile with an amplitude spectrum with $a$ value of $b$ less than $-3 / 2$. Such a profile would have a fractal dimension close to 1 , but not less than 1 . It is equally possible to produce a section with $b>-1 / 2$, but again this must have a fractal dimension $\leq 2$. This problem was addressed by Fox 
[1989]. For values of $b \geq-0.5$ the fractal dimension approached 2.0, but never exceeded 2.0. A fractal dimension of 2.0 implies a plane filling curve. This is impossible for an ordered time series, as there can only be one $y$ value for a given $x$ value. Similarly at $b \leq-1.5$ the fractal dimension never equaled 1, but asymptotically approached 1. Fox [1989] derived an empirical relationship between $b$ and $F$ which can be used to more accurately determine $F$ from $b$. Unfortunately, there are additional problems with using the spectral method. First, spectra derived from real data are often very noisy [e.g. Turcotte, 1992, p. 82], and long sections are required to find a reasonably stable value of $b$. Second, there is a particular problem in removing the average dip from faulted sections.

The amplitude spectrum of a sloping straight line (e.g. a triangle function or ramp) is power-law with $b=-1.0$. [e.g. Kreyszig, 1988, p. 599]. Applying $F=5 / 2+b$, would give a $F=1.5$, using the results of Fox [1989] gives $F=1.47$. A simple sloping line actually has a fractal dimension $F$ of 1.0 . Any profile with an average slope will give a power law spectrum with $b \sim-1.0$, irrespective of the true fractal properties. To avoid this problem it is necessary to remove any average slope, or regional dip with respect to horizons. This is common practice, however Weissel et al. [1994] suggest that such de-trending may alter the underlying scaling properties, as it has a disproportionate effect on the topography at larger length scales. More importantly, if a section has two average slopes, they cannot both be removed prior to analysis. A section with a set of normal faults has two significant slopes within it, the regional dip of the horizon and the average dip of the large fault planes. If an attempt is made to remove one dip, then the other will remain. This leads to the spectra of these sections giving values of $b$ close to -1.0 , irrespective of the true roughness. Therefore this method has not been used for the sections in this paper.

\section{Analysis of Roughness of Simulated Faulted Horizons}

The true roughness of a faulted horizon will not only depend on the offsets caused by the faults, but also on the physical roughness of the fault planes and the horizon itself. Here the assumption is made that the roughness of the fault plane and the horizon is of significantly smaller magnitude than that produced by the fault offsets and is therefore neglected. A simple model is used of a horizontal surface, offset by parallel normal faults with faults down-throwing in either direction with equal probability. A vertical section is then taken through this horizon, perpendicular to the strike of the faults. Within the model there are several parameters that will determine the fractal dimension. These include the fault displacement population, the positioning of the faults, and fault dip. The aim is to model continentâl and non-ridge related oceanic fault populations which have been shown to follow a power law distribution of displacement. For consistency with Pickering et al. [1995] the cumulative definition is used to define the fault displacement population:

$$
N=c u^{-D}
$$

where $N$ is the number of faults $\geq u$. Models for the positioning of faults are less well developed, and are frequently based on the distribution of the spacings between faults along dip sections. Resolution limited sections cannot reliably differentiate between different spacing populations, as the spacings between the visible faults are not drawn from the true underlying spacing distribution. Studies of faulting at outcrop where the minimum resolution is closer to the real limit of the fault population, have suggested a clustered distribution rather than a uniform one, but whether this is closer to negative exponential or power law is still not clear [e.g. Gillespie et al. 1994]. Line et al. [1997] find a negative exponential distribution for a fault set cutting dolerite sills in central Sweden.

The dip of normal faults varies from close to vertical. down to $30^{\circ}$ or less depending on the depth of the fault within the crust and the detailed mechanics of the faulting [Jackson and McKenzie, 1983]. Within a simulation, the effect of each parameter on the fractal dimension can be quantified and tested for statistical significance. In each simulation run the section has the same statistical properties; however, the order of the faults on the section and the spacings between them are picked at random from the chosen distributions.

\subsection{Varying the displacement population $D$ value}

The effect of the $D$ value of the fault displacement population on $F$ was analyzed using synthetic sections with vertical faults with a maximum (arbitrary) displacement of 3.0 and $D$ values varying from 0.5 to 2.0 at intervals of 0.1 . Example sections are shown in Figure 1. The scale range of the sections was held constant by varying the number of faults, for example, at $D=2.0,10,000$ faults were required, whereas at $D=1.0$ only 100 faults were used. The scale range of the fault population on each section was held constant because, as demonstrated in Figure 2, varying the resolution limits for a constant $D$ value of the fault population can cause a variation in the measured fractal dimension. The estimates of $F$ from the dispersion methods remain relatively constant until the population is reduced to $\sim 100$, below which the values rapidly decrease. The length estimator method is more sensitive to the scale range limitations of the data, with $F$ values falling below a sample size of $\sim 300$. In order to remove this effect from the simulation results the scale range of the population must be constant. Two orders of magnitude were chosen to be analogous to the typical scale range on seismic sections, and 10,000 was the limit for the largest number of faults that could be used. The faults were positioned randomly, producing a negative exponential

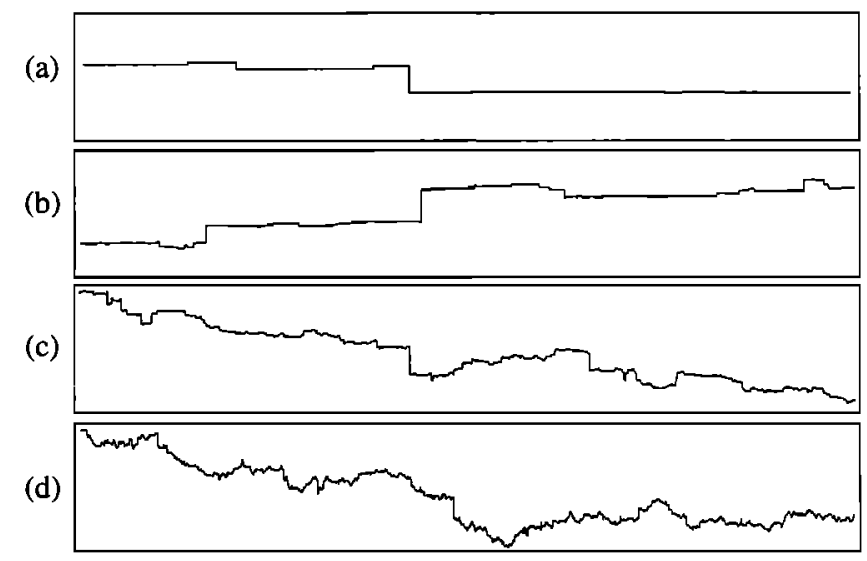

Figure 1. Examples of the sections produced and analyzed in the simulation. The faults are all vertical and follow a power law distribution of displacement with a constant scale range. The spacing population for these examples is negative exponential. The faults face in either direction with equal probability. The $D$ value of the population and the number of faults in each example are (a) $0.5-10$, (b) $1.0-100$, (c) 1.5 1000 and (d) 2.0 - 10,000. The horizontal and vertical scales are arbitrary and make no difference to the fractal dimension of the self-affine scaling of each section. 


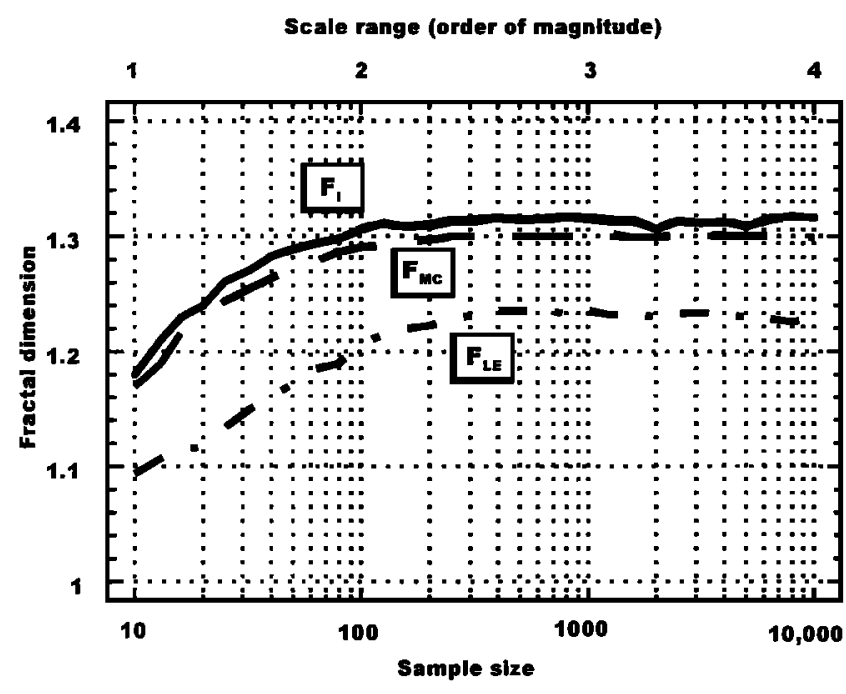

Figure 2. Graph showing the effect of truncating the fault population on the measured fractal dimension. All the sections analyzed had displacement populations with $D=1.0$ and a negative exponential spacing set. The scale range of the faults was changed by varying the number of faults while keeping the maximum fault size constant. All three $F$ measures are reduced by truncating the population, particularly at scale ranges lower than two orders of magnitude.

distribution of spacings with an average spacing $\langle s\rangle$ which varied so that all the sections were $\sim 100$ arbitrary units long. Varying the maximum fault size (and $\langle s\rangle$ as will be discussed later) will have no effect on the fractal dimension $(F)$. At least 1,000 sections were produced for each $D$ value. Each one was sampled producing a series of 512 values, which were then analysed using the methods described earlier.

Figure 3 shows an example of the typical graph produced for each analysis method for a section produced from a $D$ value of 1.0. These graphs show a good fit to the single fractal model. At each population $D$ value, each set of values of $F_{l}$, $F_{M C}$, and $F_{L E}$ form an approximately normal distribution (Figure 4). The spread of values may be caused by measurement error, if all the sections have the same theoretical value of $F$. Alternatively, it may represent a real spread in the actual values of $F$ due to the varying positions of the faults and spacings. It is most likely that the spread is a combination of the two, although in what proportion is difficult to quantify. The mean values of $F$ derived for each $D$ value are plotted on Figure 5 together with the associated standard deviations.

As expected, there is an increase in fractal dimension $F$, with increasing population $D$ value (Figure 5). At low population $D$ values $(\leq 0.5), F$ is close to 1.0 and the sections are very smooth. As the fault population $D$ value increases above 0.5 , the fractal dimension measured by all the methods increases, however, the average value of $F_{L E}$ is slightly lower than that of the two dispersion methods for $D$ values less than 1.5. This difference is only marginally significant, and is likely to be due to the slightly greater sensitivity of this analysis method to the scale limitations of the fault set. At high $D$ values the fractal dimension measured by all the methods approaches $\sim 1.5$. This is in broad agreement with the results of Malinverno and Cowie [1993], who found a fractal dimension of 1.5 for a fault population obeying a negative exponential frequency distribution. Scale-limited fault sets from such a population are similar to scale-limited sets from a power law distributed population with a high $D$ value.

\subsection{Varying the dip of the faults}

The effect of fault dip on the fractal dimension of the surface was investigated in a series of simulations in which dips were varied at regular intervals from $30^{\circ}$ to $90^{\circ}$. In these simulations the fault displacement population was power law with $D=1.0$, the spacing distribution was negativeexponential and each section had 100 faults, with a maximum displacement of 3.0. For each simulation, 1,000 sections were produced and analyzed.

The results are shown in Figure 6 and indicate that the dip of the faults makes little difference to the values of either $F_{I}$ or $F_{L E}$. In contrast the values of $F_{M C}$ decrease with decreasing dip which is likely to be caused by the calculation method. The $F_{M C}$ method calculates $\sigma$ based on the deviations from a line fitted using a least-squares criteria. When the faults are vertical, the fitted line will never fall on a fault plane as any sub-section will always partly include some horizon (Figure 7a). For dipping faults it is possible for a sub-section to only contain a fault plane, in which case the total deviation for that sub-section will be zero (Figure $7 \mathrm{~b}$ ). As this is more likely to occur the shorter the sub-section taken, the average dispersion measured for these short sub-sections will decrease. The effect on the $\sigma-l$ graph is to cause an increase in gradient at smaller lengths giving a greater average slope. This increases the measured Hurst exponent and decreases the measured fractal dimension. As this effect is more likely to occur at lower angles of dip, the values of $F_{M C}$ decrease with decreasing dip. Although the values of $F_{M C}$ are systematically lower, the differences are small and are related to the method of measurement rather than any real physical change in the roughness of the surface.

\subsection{Varying the spacing of the faults}

The fractal dimension of the faulted surface may be affected by the positioning of the faults on the section. Two representative statistical spacing distributions were tested in the simulations, a negative exponential distribution, and a power-law distribution. The profiles described so far have all had a random positioning of faults, that is a negative exponential distribution of spacings. These were defined by an average spacing $\langle s\rangle$. It is important to appreciate that reducing $\langle s\rangle$ does not increase the clustering, it only increases the fault density. Reducing $\langle s\rangle$ will also reduce the length of the section for a given number of faults, and therefore each fraction of the section will still contain as many faults as it did before, and the estimates of $F$ will remain the same.

The clustering can be increased by using a power-law distribution of spacings, defined by exponent $S$. As $S$ is decreased, the set of spacings decrease more rapidly with cumulative number, and the faults are positioned closer together. Therefore spacing populations with lower values of $S$ are more clustered than those with high values of $S$. Simulations were made using a series of values of $S$, ranging from 0.25 to 2.0 , at intervals of 0.25 . The maximum spacing was set at 20 for all the simulations. Varying the maximum spacing will not affect the fractal dimension, for reasons similar to those discussed for varying $\langle s\rangle$. The scale range of the spacing set will depend on the value of $S$ (for a fixed number of faults), as the displacement range depended on $D$. Therefore, high values of $S$ will give a narrow scale range of spacings. The results of this simulation are shown in Table 1.

For high values of $S$, there is little difference from the values calculated for the negative-exponential spaced sections. This is expected, as the resolution limited spacing sets produced by high values of $S$ will be similar to the negative-exponential set of spacings. For the dispersion methods, $F_{I}$ and $F_{M C}$, the values decrease as $S$ decreases. This 
(a)
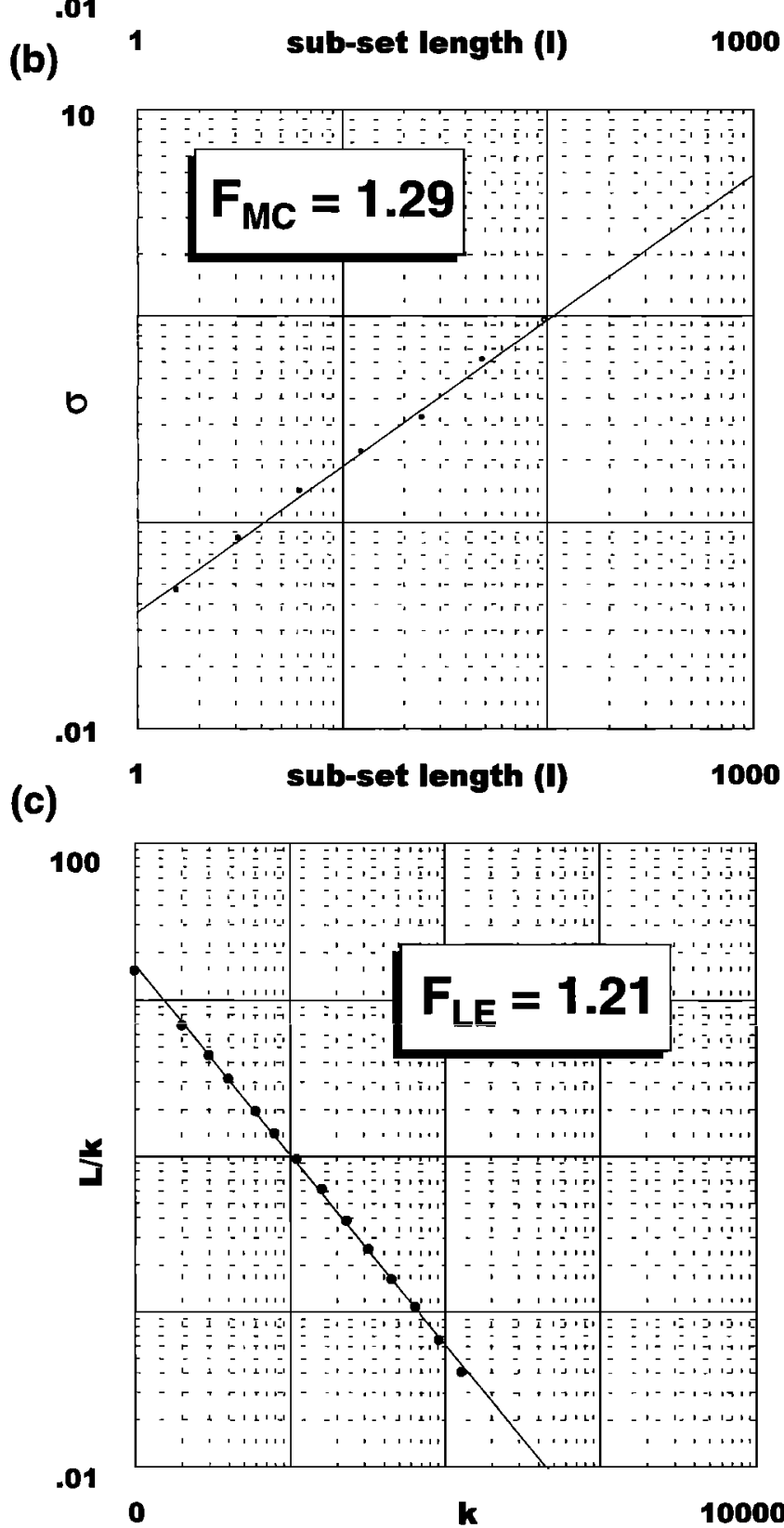

can be understood by considering how $\sigma$ is calculated. The values of $\sigma$ used for the graphs are the average value of $\sigma$ from all the sub sections at that particular length. A large fraction of a section with clustered faulting will contain similar numbers of faults as the same fraction of a section with more uniformly distributed faulting. These will therefore give similar values of $\sigma$. In contrast, the majority of small fractions of a clustered section will contain no faults at all, as they will occur between clusters of faults and give zero values of $\sigma$, reducing the average value of $\sigma$ measured at small length scales. This will steepen the $\sigma-l$ graph, increasing $H$ and decreasing $F$. The values of $F_{L E}$ show a different trend. There is a small increase in $F_{L E}$ from 1.22 at $S=2.0-1.5$, to 1.27 at $S=0.5$. The increase is accompanied by an increase in the spread of values and may not be significant, but the values of $F_{L E}$ do not decrease. This is again due to the calculation method. This method does not split the sections into small fractions, but examines a series sampled at different interval sizes. The majority of the curve length will be contributed by intervals which include jumps across faults. Therefore the curve length is mainly controlled by the size and number of faults, but is relatively insensitive to the spacing.

\section{Analysis of Roughness of Natural Faulted Horizons}

Several factors need to be considered when relating the fractal dimension of a natural faulted horizon to the $D$ value of the fault population. Industry standard seismic sections only resolve the larger faults (usually $10 \mathrm{~m}$ or more of throw) and cover 2 orders of magnitude at best. This results in a poorly defined $D$ value for individual sections. The fault population may also not be ideally power law: although the power law model is the best fitting distribution, individual sections will vary and may have more large (or small) faults than predicted by the ideal model. Therefore the variation in roughness may be greater for sections with the same fault population $D$ value than in the simulation. The simulations do not model the response of horizons to faulting (drag and roll over, for example) and this may produce more roughness than that predicted by the simulation.

\subsection{SSL-MF89 Moray Firth Seismic Sections}

These sections are derived from the SSL-MF89 survey from the Moray Firth. The fault displacement population in the Moray Firth has been established previously as power-law by Pickering et al. [1994]. There are 21 sections from this set which contain sufficient faults to cover the two orders of magnitude required to avoid resolution effects in the

Figure 3. Graphs used to find the values of $H$ and $F$ for a typical simulated section. The section contained 100 faults, following a power law displacement distribution with $D=$ 1.0 , and a negative exponential spacing set (i.e., similar to Figure 1b). All three graphs show a good fit to a straight line, confirming that the sections are fractal. (a) Graph of $\sigma$ against sub set length $(l)$ calculated using the internal dispersion method of Ivanov [1994]. The gradient of a line fit using a least squares method gives a Hurst exponent $H_{I}=0.69$, hence $F_{I}=2-H_{I}=1.31$. (b) Graph of $\sigma$ against $l$ calculated using the deviation from a best fit line as used by Malinverno and Cowie [1993]. The graph gives $H_{M C}=0.71$, hence $F_{M C}=1.29$. (c) Graph of $L / k$ against $k$ where $L$ is the curve length calculated using the method of Higuchi [1988] and $k$ is the interval size. The graph yields $F_{L E}$ directly, which in this case is 1.21 . 


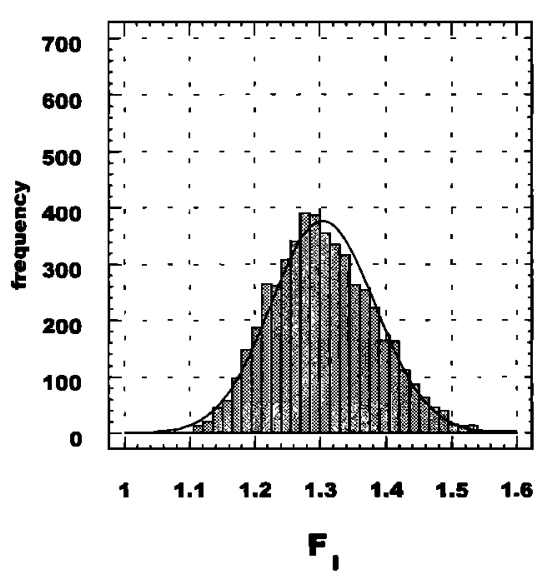

(a)

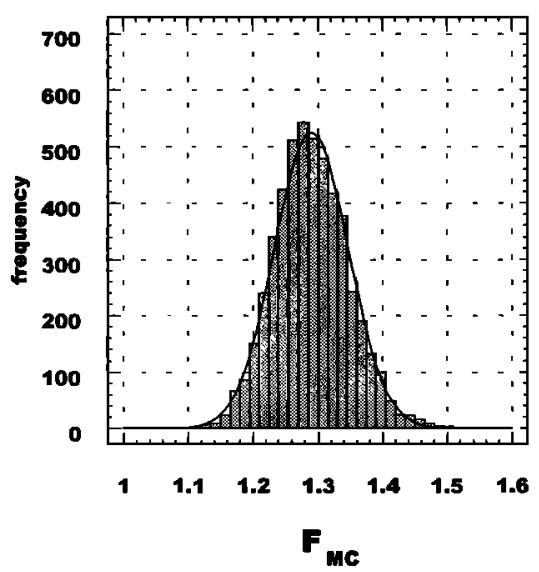

(b)

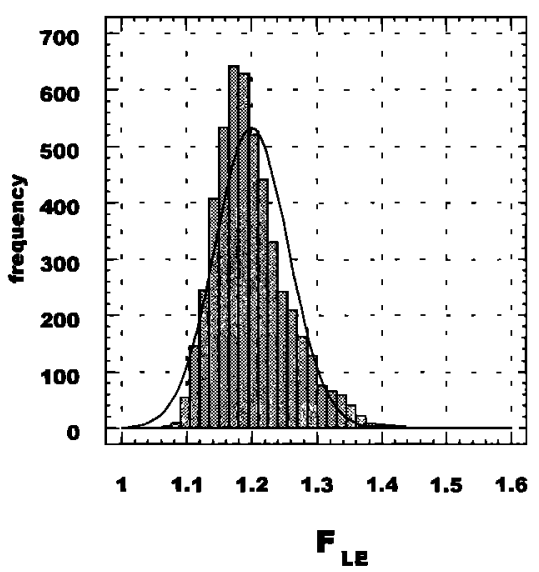

(c)

Figure 4. Histograms of the measured values of (a) $F_{I}$ (b) $F_{M C}$ and (c) $F_{L E}$, for 5,000 simulated sections. Each section contained 100 faults, following a power-law distribution of displacement with $D=1.0$. The spacings followed a negative-exponential distribution. Each set of $F$ values approximately fit a normal distribution (also shown) with (a) mean $=1.30$ and standard deviation 0.08 , (b) $1.29,0.06$ and (c) $1.20,0.06$. The means and standard deviations for a series of these distributions produced by varying the $D$ value of the fault population, are shown in Figure 5.

estimation of surface roughness. The horizon chosen for analysis was the Top Triassic as this is a clear reflector and was also used in Pickering et al. [1994]. An example section is shown in Figure 8. The average $D$ value for the set of seismic profiles is 0.84 , while individual sections have best fitting power-law distributions with $D$ values which vary from 0.63 to 1.14 . Although the population on each section is likely to be a random sample from a fault population of $D \sim 0.8$, the $D$ values for the individual sections may more closely relate to the surface roughness, as they better represent the variations in faulting between the sections. The spacing distributions were not well-defined, although most of the sections gave a reasonable fit to a negative exponential distribution and none of the sections had faults that were particularly clustered.

Each section was sampled into a series of 512 values ( every $75 \mathrm{~m}$ ) and then analyzed to find $F_{l}, F_{M C}$ and $F_{L E}$. Typical graphs for the methods are shown in Figure 8. The graphs show a good fit to the single fractal model, which precludes any use of more complex multi-scaling analyses.
The results from all the analyses are summarized in Figure 9, with the estimates of $F$ plotted against the $D$ value derived from the fault set on each section. Also plotted are the results from a series of simulations run with parameters designed to match those of the Moray Firth sections. These simulations were run with fault displacement population $D$ values from 0.6 to 1.2. The scale range of the fault set was kept constant at 2 orders of magnitude, a negative exponential (random) spacing was used in the simulations together with a fault dip of $70^{\circ}$ (the average dip of the faults on the SSL-MF89 sections). The large variation in the $F$ values given similar $D$ values means that a single section alone cannot be used to accurately predict population $D$ values. However, there is agreement between the measured and predicted averages of $F$ and $D$.

Figure 9a shows the results using the $F_{I}$ method. Most of the values of $F_{1}$ fall within the expected range from the simulation. As this is only the center $68 \%$ of the distribution, five or six values in a sample of 21 would be expected to lie
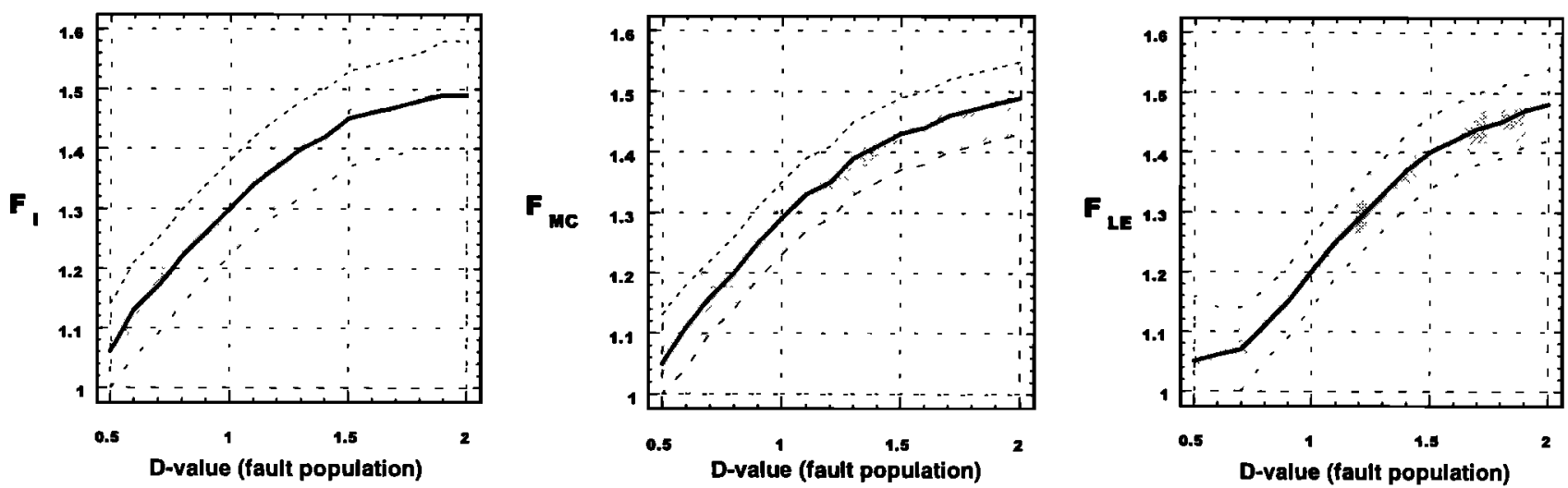

Figure 5. Plot of the average (a) $F_{l}$, (b) $F_{M C}$ and (c) $F_{L E}$ for sections with fault displacement population $D$ values varying from 0.5 to 2.0 . The number of faults was varied from 10 to 10,000 to hold the scale range of the fault set constant at 2 orders of magnitude. The spacing distribution was negative exponential. The mean value of $F$ for each set of sections is shown as a solid line. The dotted lines are the mean plus or minus the standard deviation of the set of $F$ values at each population $D$ value. The values of $F$ increase from $\sim 1$ at $D=$ 0.5 to $\sim 1.5$ at $D=2.0$. 


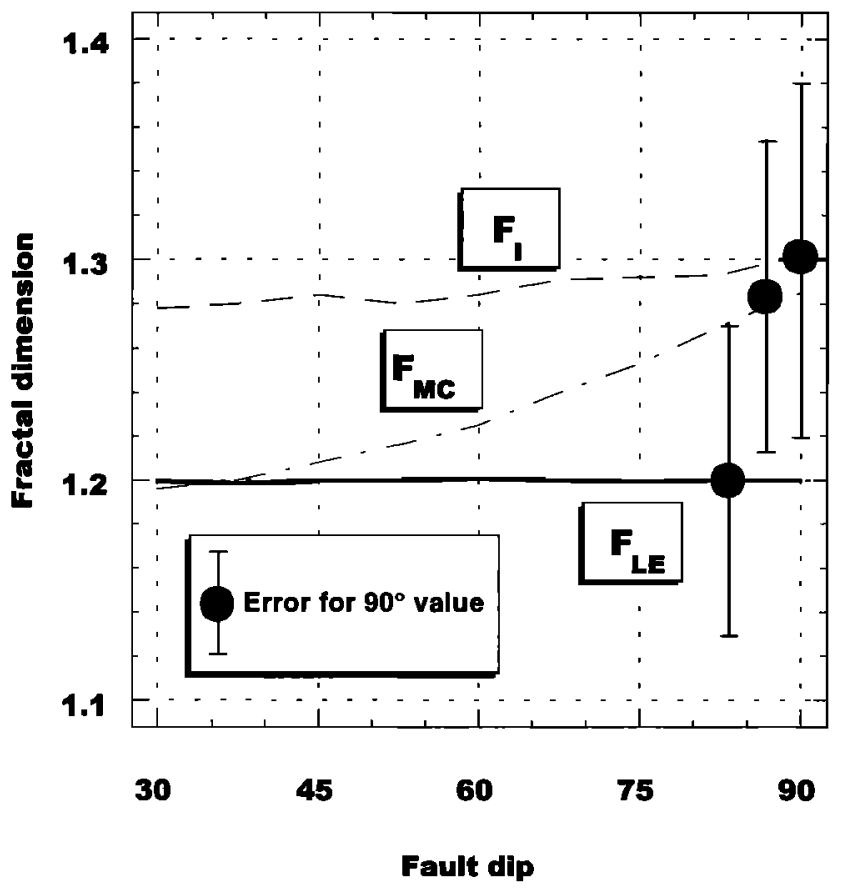

Figure 6 Graph showing the effect of fault dip on the measured fractal dimension. All the sections contained 100 faults, with a negative exponential distribution of spacings and a $D$ value of 1.0 . Fault dip was varied from $30^{\circ}$ to vertical. $F_{I}$ and $F_{L E}$ are not significantly affected by fault dip. In contrast $F_{M C}$ is reduced as dip is decreased (see text).

outside this band. The average value of $F_{I}=1.25$, which is close to the 1.22 predicted by the simulation for a $D$ value of 0.84 , which is the average for this set of sections. The values of $F_{M C}$ are also close to the expected band, but more fall outside than would be expected. The average value is slightly lower than predicted by the simulation at $D=0.84,1.13$ as compared to 1.16 , but the agreement is still reasonable. The lower value of this measure compared to $F_{I}$ is caused by the dip of the faults. Figure 9c shows the results of the length estimator method. The $F_{L E}$ values fall within the expected band from the simulation, but are generally slightly higher than predicted. The average value at $D=0.84$ is 1.18 , which is slightly higher than the 1.14 predicted, but again the agreement is reasonable.

\subsection{Central Indian Ocean seismic section}

The second example is from a $500 \mathrm{~km}$ multi-channel seismic reflection profile shot over the intra-plate deformation area in the central Indian Ocean [Bull and Scrutton, 1992]. The tectonics of the area have been documented in several studies [e.g. Weissel et al., 1980; Petroy and Wiens, 1989; Stein and Weissel, 1990; Neprochnov et al., 1988; Bull, 1990; Bull and Scrutton, 1990 and 1992; Chamot-Rooke et al., 1993; Van Orman et al., 1995], which have identified many structures related to deformation under a compressive stress regime. The horizon analyzed here is the lowermost sedimentary horizon that could be consistently interpreted above the top of oceanic layer 2 , which is offset by a series of reverse faults. These are interpreted as reactivated normal faults originally formed at or close to the mid-ocean ridge [Bull and Scrutton, 1992]. The fault displacement population was analyzed by Bull and Scrutton [1992] who identified two power law distributions. At the large scale $(150-900 \mathrm{~m})$ the faults obey a power law with a high $D$ value of 1.9 . Below $150 \mathrm{~m}$ the analysis is less certain, but there is a clear change in the population, with a relative drop in fault frequency. They argued that this change in distribution is either caused by the finite thickness of the strong brittle layer, or altematively is due to one of the fault sets representing a reactivation of a pre existing fabric with the other a new fault set.

Van Orman et al. [1995] analyzed a number of north-south seismic reflection profiles within the Central Indian Ocean Basin and concluded that while the average fault spacing of 7 $\mathrm{km}$ was maintained across the zone of deformation, the displacement on the faults increased from west to east (with greater distance from the convergence pole) indicating that deformation had proceeded by the reactivation of a select fault population. With continued convergence across the deformation zone the reactivated faults continued to accumulate strain while few new faults where initiated.

The fractal dimension $F$ of the horizon in Figure $10 \mathrm{a}$ was calculated using the three techniques outlined earlier and the results are shown in Figures 10b - 10d. Note that as the

(a)

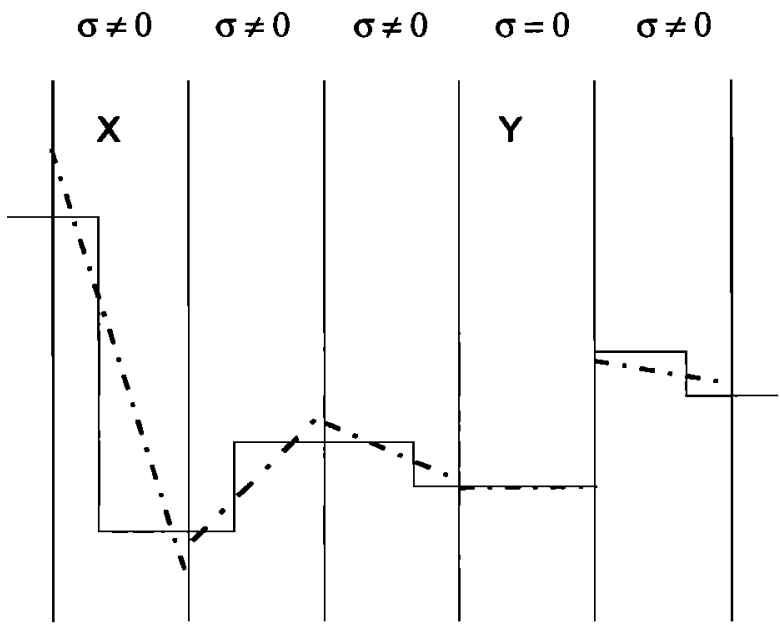

$$
\sigma=0 \quad \sigma \neq 0 \quad \sigma \neq 0 \quad \sigma=0 \quad \sigma \neq 0
$$

(b)

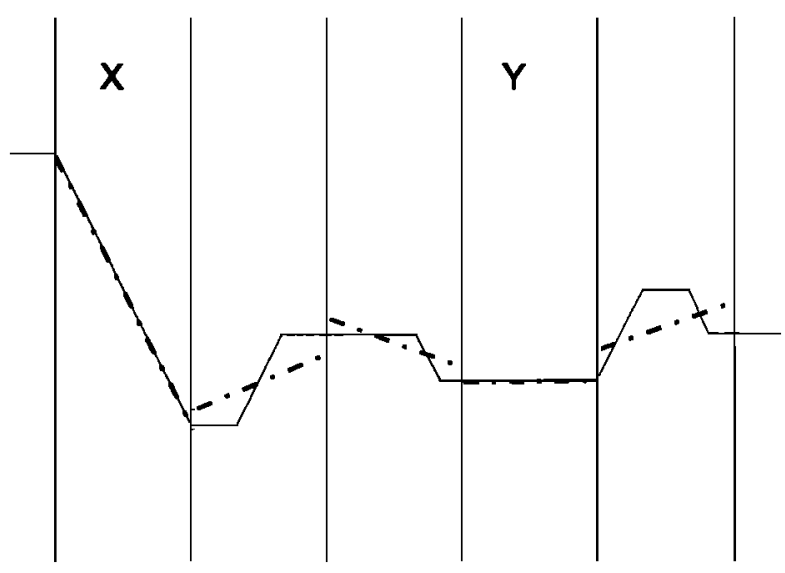

Figure 7 Schematic drawing showing how the calculation of sigma in the $F_{M C}$ method causes lower values of $F_{M C}$ when the faults have non zero dip. The solid line represents the section, and the dashed line represents the best fit line to each sub section. (a) All the faults are vertical and only sub-sections which only contain horizon (e.g. $Y$ ) will give zero values of $\sigma$. (b) Faults have non vertical dips, but most of the sub sections containing faults still give values of $\sigma \neq 0$. However, the largest fault is now entirely within sub section $X$, and this sub section gives a zero value of $\sigma$. 
Table 1. Results of the Simulation Testing Different Power Law Distribution of Spacings for the Same Fault Displacement Population.

\begin{tabular}{cccc}
\hline \multicolumn{1}{c}{$S$} & $F_{I}$ & $F_{M C}$ & $F_{L E}$ \\
\hline 0.50 & $1.20 \pm 0.13$ & $1.17 \pm 0.12$ & $1.27 \pm 0.13$ \\
0.75 & $1.26 \pm 0.09$ & $1.23 \pm 0.07$ & $1.24 \pm 0.08$ \\
1.00 & $1.28 \pm 0.08$ & $1.26 \pm 0.07$ & $1.23 \pm 0.06$ \\
1.25 & $1.30 \pm 0.08$ & $1.28 \pm 0.06$ & $1.23 \pm 0.06$ \\
1.50 & $1.31 \pm 0.08$ & $1.29 \pm 0.06$ & $1.22 \pm 0.06$ \\
1.75 & $1.31 \pm 0.08$ & $1.30 \pm 0.06$ & $1.22 \pm 0.05$ \\
2.00 & $1.32 \pm 0.08$ & $1.30 \pm 0.06$ & $1.22 \pm 0.05$ \\
\hline
\end{tabular}

In this simulation $D=1.0$ and the maximum fault size was 3.0 . 1000 sections were produced for each value of.

sampled sections must be single-valued, the dips of the faults are not honored and the faults become effectively vertical, positioned at the tip of the hanging-wall. All three graphs show a change in scaling, at length scales of around 5-6 km. This change is likely to be caused by the change in fault displacement population, where the sharp decrease in fault density has caused a reduction in the fractal dimension of the horizon. The values from each method are all fairly similar, with the large scale values of $F$ around 1.5 and the smaller scale dimension around 1.1 (see Table 2).

In order to compare these results to the simulation, the program was altered so that the apparent movement on the faults was reverse. Two fault populations were used, mirroring the results of Bull and Scrutton [1992] with a dip of $40^{\circ}$ and a negative exponential spacing distribution. The first had a $D$ value of 1.9 , and a maximum displacement of $900 \mathrm{~m}$.
The second population had a maximum at $150 \mathrm{~m}$, but the $D$ value was varied from 0.5 to 1.5 , due to the uncertainty in the measurement of this parameter. The sections were then sampled to form a single-valued section, making the faults vertical. Two fractal dimensions of the synthetic profile were derived from the graphs of each method, in the same way as for the analysis of the natural section. The dimensions at the large scale, shown in Table 2, match those from the real data quite closely, with any difference between the mean from the simulation and the measured value from the natural section well within the expected range. Note that the values from the simulation are lower than those shown in Figure 5 due to the narrower scale of the population used. The values for the fractal dimension at the small scale (see Table 2) show a more significant difference from those seen on the natural section. Even when a small scale $D$ value of 1.5 was used the values $(1.07,1.05$ and 1.01 respectively) were still below those measured, particularly $F_{L E}$. Given the uncertainty in the fault population analysis at the small scale, it is difficult to attach much significance to this difference; however, there may be an additional source of roughness, the most likely being folding associated with the reverse faulting.

In summary, for the Central Indian Ocean Basin the simulations support the contention that the fractal dimension of a faulted surface is controlled by the underlying fault displacement distribution. This change in scaling at $5-6 \mathrm{~km}$ in $F$ together with the observation of Van Orman et al. [1995] that there is no change in mean fault spacing across the convergence zone, suggests that there is a mechanical control on fault spacing. It is interesting to note that the change in scaling occurs at a length scale similar to the thickness of the oceanic crust.

(a)

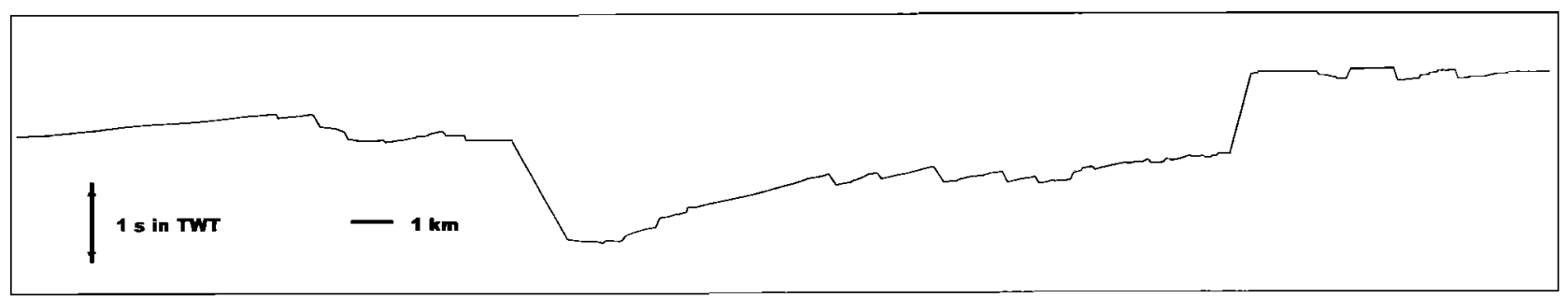

10

(s)

0.01

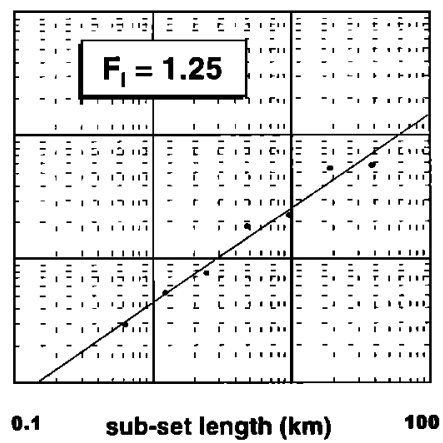

(b)

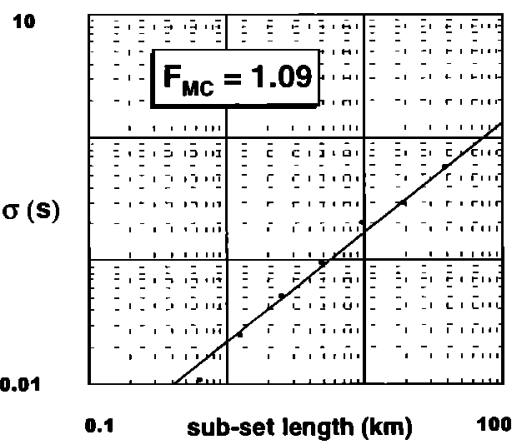

(c)

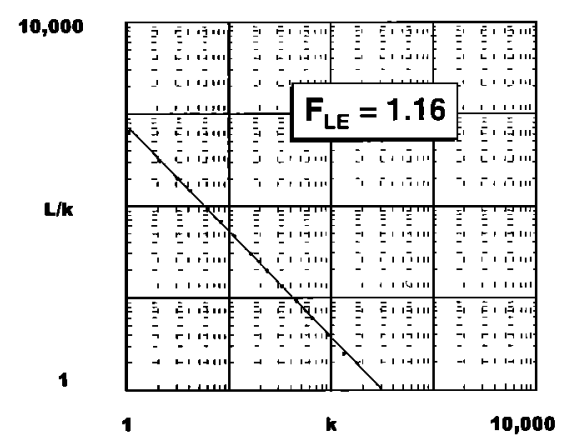

(d)

Figure 8. (a) Example horizon from one of the SSL-MF89 seismic sections. The horizon is Top Triassic, offset by a fault population with a $D$ value of approximately 0.8.(b) - (d) Graphs used to find $H$ and $F$ for the SSL-MF89 section shown in Figure 8a: Figure 8b $F_{l}$, Figure $8 \mathrm{c} F_{M C}$ and Figure $8 \mathrm{~d} F_{L E}$ (see Figure 3 ). The graphs show a reasonable fit to a straight line giving $F_{I}=1.25, F_{M C}=1.09$, and $F_{L E}=1.16$, respectively. 


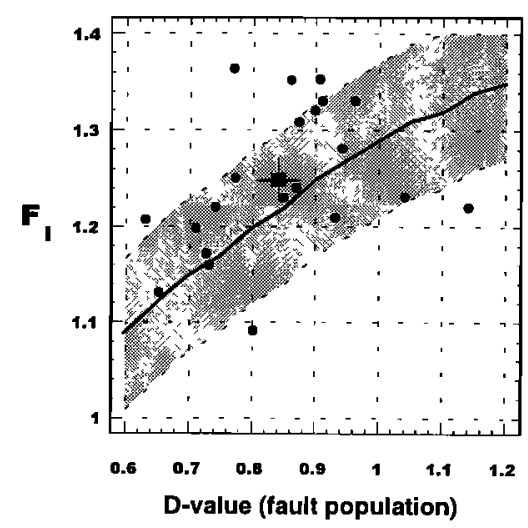

(a)

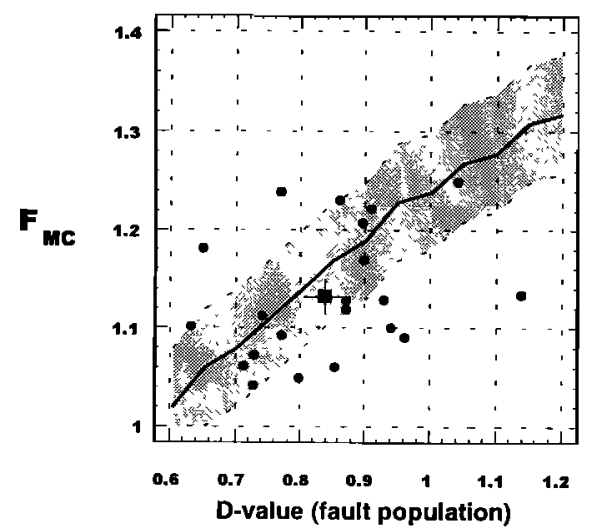

(b)

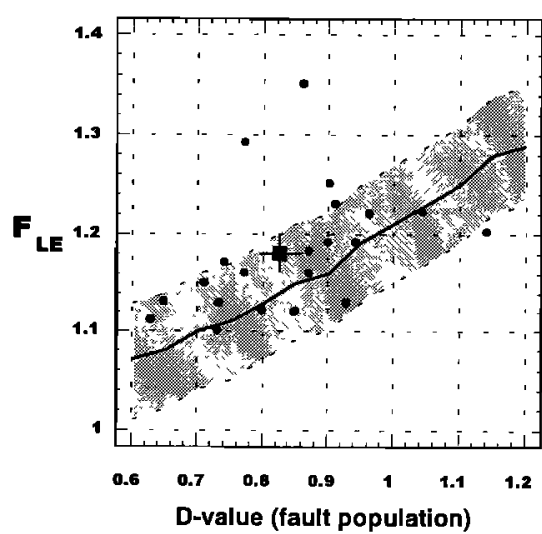

(c)

Figure 9. Plots of fractal dimensions (a) $F_{l}$, (b) $F_{M C}$ and (c) $F_{L E}$, plotted against the fault population $D$ value for each of the 21 SSL-MF89 Top Triassic horizons. The average value of each set of $F$ measures (solid squares) is plotted against the average $D$ value $(0.84)$. The error bars for this average are the standard error $(\sigma / \sqrt{ } v)$ in both $F$ and $D$. Also shown are the results of a simulation run with sections designed to match the dip and scale range of the faults on the SSL-MF89 sections (see text). These are plotted as Figure 5.

\section{Summary and Conclusions}

Three measures of the fractal dimension $F$ of a surface are outlined, two of which $F_{I}$ and $F_{M C}$ use roughness scaling methods, the other $F_{L E}$ is based on the length estimator method. A simple model of a faulted horizon was used to simulate the variation in the fractal dimension $F$ of a profile with changes in the fault population. The fault displacements were always power-law distributed, as there is considerable evidence that such a distribution is a good description of many populations, and best describes both of the natural examples used in this paper. Such a distribution can only apply over a finite range of displacement and length scales, and this range limits the application of the methods described in this paper. However as this range can spread from millimeters to kilometers (as is the case for the Moray Firth example), this does not necessarily limit the usefulness of this type of analysis.

In the simulations the horizon was originally horizontal and planar and was then offset by planar faults. A number of

(a)

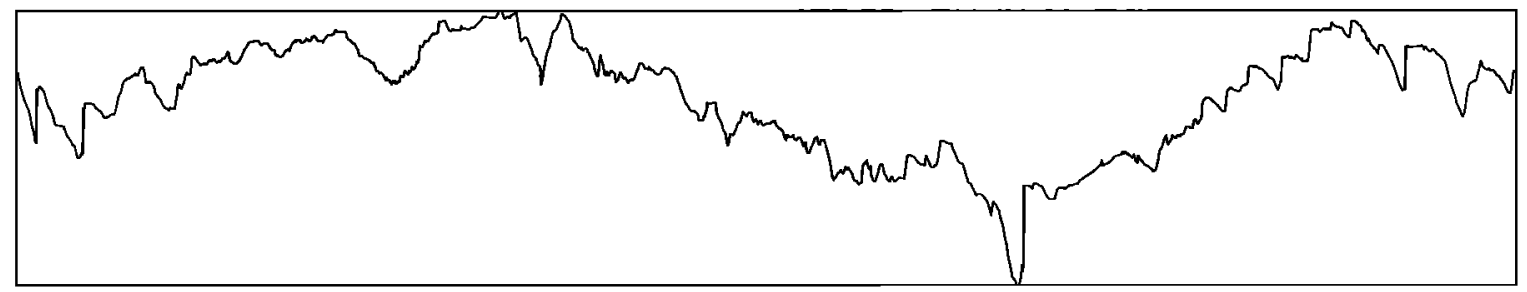

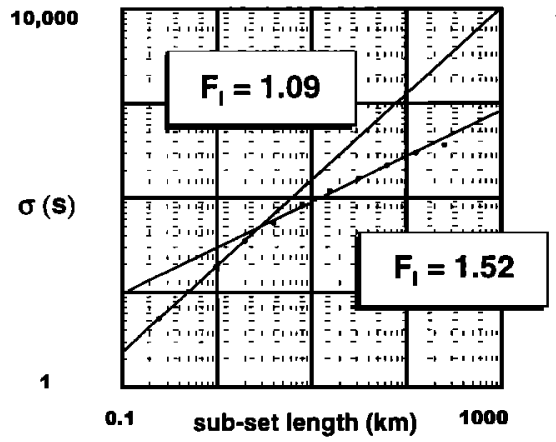

(b)

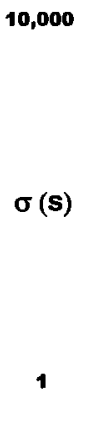

0.1

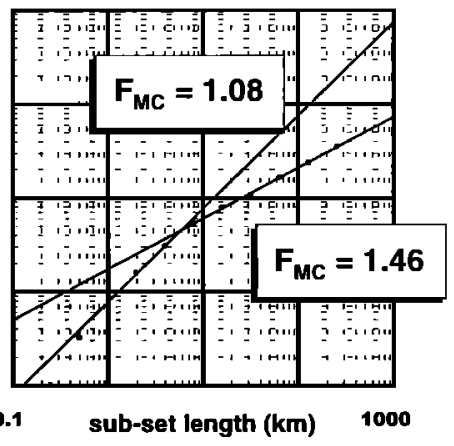

(c)

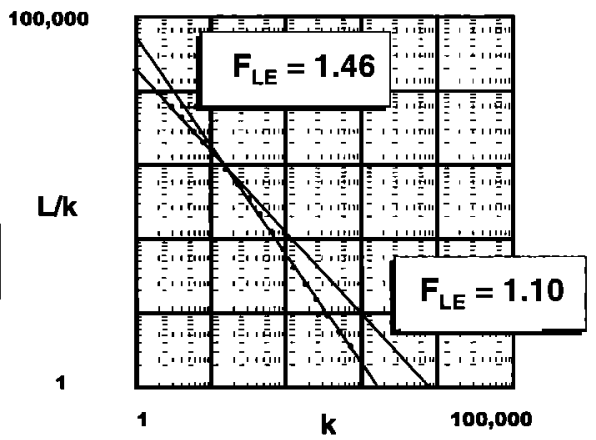

(d)

Figure 10 (a) Digitized horizon representing the loweimost sedimentary horizon that could be consistently interpreted from the north-south multi-channel seismic reflection profile described by Bull and Scrutton [1990,1992] within the Central Indian Ocean Basin.(b-d) Graphs from the three analysis methods used: Figure $10 \mathrm{~b}, F_{l}$, Figure $10 \mathrm{c}, F_{M C}$ and Figure 10d, $F_{L E}$ (see Figure 3 ). The graphs show a scaling change at $5-6$ $\mathrm{km}$, giving a lower fractal dimension at the smaller scale related to a change in the fault population (see text). 
Table 2. Comparative Results for the Simulated and Natural Indian Ocean Sections.

\begin{tabular}{lllll}
\hline & \multicolumn{1}{c}{ Sections } & \multicolumn{1}{c}{$F_{I}$} & \multicolumn{1}{c}{$F_{M C}$} & \multicolumn{1}{c}{$F_{L E}$} \\
\hline Large scale & Simulated & $1.46 \pm 0.10$ & $1.45 \pm 0.07$ & $1.39 \pm 0.10$ \\
& Natural & 1.52 & 1.46 & 1.46 \\
Small scale & Simulated & $1.06 \pm 0.05$ & $1.04 \pm 0.04$ & $1.01 \pm 0.01$ \\
& Natural & 1.09 & 1.08 & 1.10 \\
\hline
\end{tabular}

The simulation was made with a bi-fractal fault population with $D$ values of 1.9 and 1.0 , with maximum values of 900 and 150 respectively. The graphs were then analyzed to give two fractal dimensions, in the same way as the natural section was analyzed (see Figure 10).

fault population parameters were tested independently including the $D$ value of the fault displacement population (which was varied between 0.3 and 2.0 ), the distribution of the spacing set (power law with a varying exponent $S$ or negative exponential), and the dip of the faults (varied from $30^{\circ}$ to $90^{\circ}$ ).

Tests on the effect of truncation on the population showed that $F_{I}$ and $F_{M C}$ gave stable values if the scale-range of the resolved faults was $\geq 2$ orders of magnitude. $F_{L E}$ was more sensitive to the scale-range, requiring closer to 3 orders of magnitude for stable values.

The principal determining factor in the variation of $F$ was the $D$ value of the fault displacement population. $F$ increased from $\sim 1.0$ at $D$ values $\leq 0.5$, to $\sim 1.3$ at $D=1.0$, and then to $\sim 1.5$ at $D=2.0$. Variation in the dip of the faults led to only small changes in $F$, except for the values of $F_{M C}$ which were systematically lower (due to the calculation method). Changing the spacing distribution of the faults affected $F$, but only if the faults were heavily clustered. In this case, the estimates of $F_{I}$ and $F_{M C}$ decreased if the spacing distribution was power-law when compared to negative exponential, but only when the values of $S$ were less than 1.0. In contrast there was no difference in the values of $F_{L E}$.

Associating the fractal dimension of a natural faulted horizon with the $D$ value of the fault population is complicated by several factors. These include (1) the limited length of most natural sections, (2) the narrow scale range of the resolved fault population and (3) the contribution of other geological processes to the roughness. The fractal dimensions of the top-Triassic horizon on 21 sections from the Moray Firth were measured and compared to the results from the simulation. The measured values were generally within the expected range from the simulation. The average values of $F$ gave a predicted average population $D$ value close to the true average from the sections. The Indian Ocean example was more complex, due to a bi-fractal fault displacement population. This gave a scaling change in the measured fractal dimension of a faulted horizon, with different fractal dimensions at the large and small scales. Good agreement was found for the dimension at the large scale between the simulation and real sections; however, there were significant differences between the two sets of values at the small scale. This is probably due to additional sources of roughness from folding associated with the reverse faulting.

For the purpose of finding the fault displacement population $D$ value from the measured roughness, $F_{I}$ and $F_{L E}$ give the simplest relationship. The difference between $F_{I}$ and $F_{M C}$ gives a qualitative indication of the average dip of the population, with an increasing difference as the average fault dip decreases. When using these relationships, due consideration must be given to the scale-range of the resolved population, otherwise the $D$ value may be underestimated.

Acknowledgements. The authors are grateful to $\mathrm{A}$. Malinverno and J. Engeln for careful and constructive reviews. G. Pickering acknowledges a research studentship from Mobil North Sea Limited.

\section{References}

Berry, M. V., and Z. V. Lewis, On the Weirstrass-Mandelbrot fractal function, Proc. R. Soc. London., A 370, 459-484, 1980.

Brown, S.R., and Scholz, C.H., Broad bandwidth study of the topography of natural surfaces, J. Geophys. Res., 90, 12575-12582, 1985.

Bull, J. M., Structural style of intra-plate deformation, Central Indian Ocean Basin: evidence for the role of fracture zones, Tectonophysics, 184, 213-228, 1990.

Bull, J. M., and R. A. Scrutton, Fault reactivation in the Central Indian Ocean Basin and the rheology of the oceanic lithosphere, Nature, 344, 855-858, 1990.

Bull, J. M., and R. A. Scrutton, Seismic reflection images of intraplate deformation, central Indian Ocean, and their tectonic significance, J. Geol. Soc. London, 149, 955-966, 1992.

Burlaga, L. F., and L. W. Klein, Fractal structure of the interplanetary magnetic field, J. Geophys. Res., 91, 347 $350,1986$.

Chamot-Rooke, N., F. Jestin, and B de Voogd, Intraplate shortening in the central Indian Ocean determined from a $2100-\mathrm{km}$-long north-south deep seismic reflection profile, Geology, 21, 1043-1046, 1993.

Childs, C., J. J. Walsh, and J. Watterson, A method for estimation of the density of fault displacements below the limits of seismic resolution in reservoir formations, in North Sea Oil and Gas Reservoirs II. edited by A. T. Buller, pp 309-318, Graham and Trotman, London, 1990.

Cowie, P. A., A. Malinvemo, W. B. F. Ryan, and M. H. Edwards, Quantitative fault studies on the East Pacific Rise: A comparison of sonar imaging techniques, $J$. Geophys. Res., 99, 15,205-15,218, 1994.

Farr, T. G., Micro-topographic evolution of lava flows at Cima volcanic field, Mojave desert, California, J. Geophys. Res., 97, 15171-15179, 1992.

Feder, J., Fractals, Plenum Press, New York, 1988.

Fox, C. G., Empirically derived relationships between fractal dimension and power-law form frequency spectra., Pure Appl. Geophys., 131, 211 - 240, 1989.

Gillespie, P. A., C. B. Howard, J. J. Walsh, and J. Watterson, Measurement and characterisation of spatial distributions of fractures. Tectonophysics, 226, 113 - 141, 1994.

Higuchi, T., Approach to an irregular time series on the basis of the fractal theory, Physica D, 31, $277-283,1988$.

Huang, J. and D. L. Turcotte, 1989, Fractal mapping of digitized images: Application to the topography of Arizona and comparisons with synthetic images, J. Geophys. Res., 94, 7491-7495, 1989.

Ivanov, S. S., "Counter-scaling" method for estimation of fractal properties of self-affine objects. in Fractals and dynamic systems in geoscience, edited by J. H. Kruhl, PP 391-397. Springer-Verlag, Berlin, 1994.

Jackson, J., and D. McKenzie, The geometrical evolution of normal fault systems. J. Struct. Geol., 5, 471-482, 1983.

Jackson, P., and D. J. Sanderson, Scaling of fault 
displacements from the Badajoz-Cordoba shear zone, SW Spain. Tectonophysics, 210, 179-190, 1992.

Kakimi, T., Magnitude-frequency relation for displacement of minor faults and its significance in crustal deformation,. Bull. Geol. Soc. Jpn, 31, 467-487, 1980

King, G., The accommodation of large strains in the upper lithosphere of the earth and other solids by self-similar systems: the geometrical origin of b-value. Pure Appl. Geophys., 121, 761-815, 1983.

Kreyszig, E., Advanced engineering mathematics, John Wiley, New York, 1988.

Line, C.E.R., Snyder, D.B. and R.W. Hobbs, The sampling of fault populations in dolerite sills of Central Sweden and implications for the resolution of seismic data. J Struct. Geol., 19, 687-702, 1997.

Malinverno, A., Fractals and Ocean Floor Topography, A Review and Model, in Fractals in the Earth Sciences. Edited by C. C. Barton, and P. R. La Pointe, pp 107-130, Plenum, New York, 1995.

Malinverno, A., and P. A. Cowie, Normal faulting and the topographic roughness of mid-ocean ridge flanks. $J$. Geophys. Res., 98, 17921 - 17939, 1993.

Mandelbrot, B. B., Fractals: Form, Chance and Dimension., W. H. Freeman, San Francisco, 1977.

Mandelbrot, B. B., Self affine fractals and fractal dimension. Phys. Scr., 32, 257-260, 1985.

Marrett, R., and R. W. Allmendinger, Estimates of strain due to brittle faulting: Sampling of fault populations, J. Struct. Geol., 13, 735-738, 1991.

Neprochnov, Y. P., O. V. Levchenko, L. R. Merklin, and V. $\mathrm{V}$. Sedov, The structure and tectonics of the intra-plate deformation area in the Indian Ocean, Tectonophysics, 156, 89-106, 1988.

Petroy, D. E., and D. A. Wiens, Historical seismicity and implications for diffuse plate convergence in the northeast Indian Ocean, J. Geophys. Res., 94, 10613-10623, 1989.

Pickering, G., J. M. Bull, D. J. Sanderson, and P. V. Harrison, Fractal Fault displacements: a case study from the Moray Firth, Scotland, in Fractals and Dynamic Systems in Geoscience. edited by J. H. Kruhl. PP 105-119, Springer-Verlag, Berlin,1994.
Pickering, G., J. M. Bull, and D. J. Sanderson, Sampling power-law distributions. Tectonophys., 248, 1-20, 1995.

Pickering, G., D. P. Peacock, D. J. Sanderson and J.M. Bull, Modelling tip zones to predict the throw and length characteristics of faults, AAPG Bull., 81, 82-99, 1997.

Scholz, C. H., and P. A. Cowie, Determination of the total strain from faulting using slip measurements, Nature, 346 , 837-839, 1990.

Schulz, M., M. Mudelsee, and T. C. W. Wolf-Welling, Fractal analysis of Pleistocene marine oxygen isotope records, In Fractals and Dynamic Systems in Geoscience., edited by $\mathrm{J}$. H. Kruhl. pp 377-387, Springer-Verlag, Berlin 1994.

Stein, C. A., and J. K. Weissel, Constraints on Central Indian Ocean Basin thermal structure from heat flow, seismicity and bathymetry, Tectonophysics, 176, 315-332, 1990.

Turcotte, D. L., Fractals and chaos in geology and geophysics, Cambridge University Press, Cambridge, 1992.

Van Orman, J., J. R. Cochran, J. K. Weissel, and F. Jestin, Distribution of shortening between the Indian and Australian plates in the central Indian Ocean, Earth Planet. Sci. Lett., 133, 35-46, 1995.

Walsh, J. J., J. Watterson, and G. Yielding, The importance of small scale faulting in regional extension, Nature, 351, 391-393, 1991.

Weissel, J. K., R. N. Anderson, and C. A. Geller, Deformation of the Indo - Australian plate, Nature, 287, 284-291, 1980.

Weissel, J. K., L. F. Pratson, and A. Malinverno, The length-scaling properties of topography, J. Geophys. Res., 99, 13997 - 14012, 1994.

J. M. Bull (corresponding author) and D. J. Sanderson, School of Ocean and Earth Science, Southampton Oceanography Center, University of Southampton, Southampton SO14 3ZH England, UK. (email: bull@soton.ac.uk)

G. Pickering, Ikoda Ltd, 5 Old Lodge Place, Twickenham, TWI 1RQ, UK. (e-mail: giles@ikoda.demon.co.uk)

(Received December 2, 1997; revised June 2, 1998; accepted August 11, 1998.) 